\title{
Rotational Diffusion of Membrane Proteins in Crowded Membranes
}

\author{
Javanainen, Matti
}

2020-04-16

Javanainen , M , Ollila , O H S \& Martinez-Seara , H 2020 , ' Rotational Diffusion of

Membrane Proteins in Crowded Membranes ' , Journal of Physical Chemistry B , vol. 124 , no. 15 , pp. 2994-3001 . https://doi.org/10.1021/acs.jpcb.0c00884

http://hdl.handle.net/10138/328171

https://doi.org/10.1021/acs.jpcb.0c00884

acceptedVersion

Downloaded from Helda, University of Helsinki institutional repository.

This is an electronic reprint of the original article.

This reprint may differ from the original in pagination and typographic detail.

Please cite the original version. 


\title{
Rotational Diffusion of Membrane Proteins in Crowded Membranes
}

\author{
Matti Javanainen, ${ }^{*},+\ddagger$ O. H. Samuli Ollila, $₫$ and Hector Martinez-Seara ${ }^{*} \dagger$ \\ $\dagger$ Institute of Organic Chemistry and Biochemistry, Czech Academy of Sciences, Prague, \\ Czech Republic \\ $\ddagger$ Computational Physics Laboratory, Tampere University, Tampere, Finland \\ IInstitute of Biotechnology, University of Helsinki, Helsinki, Finland \\ E-mail: matti.javanainen@gmail.com; hseara@gmail.com
}




\begin{abstract}
Membrane proteins travel along cellular membranes and reorient themselves to form functional oligomers and protein-lipid complexes. Following the Saffman-Delbrück model, protein radius sets the rate of this diffusive motion. However, it is unclear how this model — derived for ideal and dilute membranes — performs under crowded conditions of cellular membranes. Here, we study the rotational motion of membrane proteins using molecular dynamics simulations of coarse-grained membranes and 2dimensional Lennard-Jones fluids with varying levels of crowding. We find that the Saffman-Delbrück model captures the size-dependency of rotational diffusion under dilute conditions, whereas a stronger scaling laws arise under crowding. Together with our recent work on lateral diffusion, our results reshape the description of protein dynamics in their native membrane environments: the translational and rotational motion of proteins with small transmembrane domains is rapid, whereas larger proteins or protein complexes display substantially slower dynamics.
\end{abstract}

\title{
Introduction
}

For membrane proteins to form protein-lipid assemblies, functional dimers, and higherorder oligomers, ${ }^{1-4}$ not only do they need to find each other via lateral diffusion, but also to locate the proper oligomerization interfaces via rotational diffusion. ${ }^{5,6}$ The Saffman-Delbrück (SD) $\operatorname{model}^{7,8}$ derived for a simplified membrane model with a lone protein suggests that the rotational diffusion coefficients $\left(D_{\text {rot }}\right)$ of membrane-embedded objects such as proteins, measured with respect to the axis normal to the membrane, depend on protein radii $R$ as

$$
D=\frac{k_{\mathrm{B}} T}{4 \pi h \mu_{\mathrm{m}} R^{2}}
$$

where $h$ is membrane thickness and $\mu_{\mathrm{m}}$ the membrane viscosity. The SD model is valid for objects smaller than the SD length, $L_{\mathrm{SD}}=h \mu_{\mathrm{m}} \times\left(2 \mu_{\mathrm{f}}\right)^{-1}$, where $\mu_{\mathrm{f}}$ is the viscosity of the solvent surrounding the membrane. More complex extensions of Eq. (1) that capture 
the diffusion coefficients of objects of arbitrary sizes have also been derived. ${ }^{9-11}$ Notably, for large inclusions — such as micrometer-sized lipid domains in gel-phase ${ }^{10}$ — a stronger scaling of $D_{\text {rot }} \sim R^{-3}$ is expected. ${ }^{10,11}$ Besides predicting diffusion coefficients, the SD model is also used to derive protein sizes ${ }^{12}$ and membrane viscosities ${ }^{13}$ from diffusion data, and to estimate the size limitations for membrane proteins in solid-state NMR experiments. ${ }^{14}$

However, cellular membranes are extremely crowded ${ }^{15}$ and contain often less than hundred lipids per protein. ${ }^{16}$ This indicates that proteins undergo repeated collisions with other proteins, ${ }^{17}$ which can further affect lipid motion via confinement and excluded area effects, indicating that essentially no "bulk" lipids exist in cellular membranes. ${ }^{18,19}$ This picture is drastically different from the one considered in the derivation of the SD model, hinting that the model should be put to test also under crowded conditions.

The slowdown of protein rotational diffusion upon crowding has been experimentally observed for bacteriorhodopsin in model membranes using fluorescence microphotolysis, ${ }^{12}$ and for sarcoplasmic reticulum Ca-ATPase, ${ }^{20}$ rhodopsin, ${ }^{21,22}$ and yeast cytochrome oxidase ${ }^{23}$ using saturation transfer electron paramagnetic resonance (STEPR). The most realistic crowding conditions can be found in living cells, but the data is scarce because measurements of rotational diffusion with different crowding levels from cellular membranes is challenging. Fluorescence and photodichroism measurements on living cells gave very different rotational diffusion coefficients of $0.25-1 \times 10^{5} \mathrm{rad}^{2} / \mathrm{s}$ for rhodopsin ${ }^{24}$ and $50 \times 10^{5} \mathrm{rad}^{2} / \mathrm{s}$ for $\alpha_{2 A}$ adrenergic receptors ${ }^{25}$ respectively. The difference in these values is quite substantial considering that both of these proteins are similarly-sized G protein-coupled receptors. This discrepancy could arise either from the specific interactions of the proteins in the complex cellular environment or from the shortcomings of experimental approaches. ${ }^{25}$ Notably, the values from two independent STEPR experiments for rhodopsin in model membranes, $0.3-1 \times 10^{5} \mathrm{rad}^{2} / \mathrm{s}^{21,22}$ agree well with the aforementioned values obtained from living cells. ${ }^{24}$ Additionally, NMR spin relaxation experiments can detect rotational dynamics of membrane proteins, but additional models are needed to determine rotational diffusion coefficients around membrane 
normal because the detected chemical bonds may not rotate in parallel to the membrane plane. ${ }^{14,26,27}$

Computationally, the rotational diffusion of synthetic inclusions in lipid bilayers was evaluated first using dissipative particle dynamics simulations. ${ }^{28}$ They revealed that the SD description agrees well with simulation data for inclusions whose size differs by multiple orders of magnitude. Also in more recent coarse-grained molecular dynamics simulation study, the rotational diffusion coefficients with different protein sizes were in reasonable agreement with the SD model. ${ }^{29}$ Notably, both of these studies were performed on singleprotein/inclusion systems, i.e. under infinitely diluted conditions. On the other hand, our recent coarse grained MD simulations demonstrated that the weak dependence $D_{\text {lat }} \sim$ $\ln \left(R^{-1}\right)$ predicted by the SD model for lateral translational diffusion holds true only in dilute conditions, but it gets replaced by a stronger Stokes-like scaling $\left(D_{\text {lat }} \sim R^{-1}\right)$ under crowded conditions. ${ }^{30}$ This finding has profound effects on protein oligomerization as larger multipass proteins move relatively slowly at the cellular scale, whereas their smaller single-pass counterparts diffuse rapidly along the membrane plane.

Therefore, it is worth asking whether the SD model for rotational diffusion also breaks down under crowded conditions. If it did, we would have to further reshape our view on protein motion in cellular membranes; specific protein-protein and protein-lipid interactions only take place when the constituents are in each others' vicinity and in a favorable orientation. Therefore, many functions of these proteins are likely diffusion-limited in terms of translation and rotation, ${ }^{5,6}$ yet their current theoretical description is based on the SD model, hence completely neglecting the effects of crowding. Moreover, the failure of the SD model under crowding would also set limitations on conditions where it can be employed to resolve properties of lipid membranes and proteins, ${ }^{12,13}$ and guide the design of NMR experiments that are limited to sufficiently fast rotations. ${ }^{14}$ 


\section{Methods}

We use coarse grained (CG) molecular dynamics simulations of membranes and simple 2dimensional Lennard-Jones (LJ) fluids to analyze the influence of crowding on rotational motion of membrane proteins. Our ultimate aim was to check if the SD model for rotational diffusion is applicable under crowded conditions. To this end, we calculated the rotational diffusion coefficients for numerous transmembrane proteins of various radii from CG and LJ simulations in differently crowded environments. The four types of simulated systems considered are schematically presented in Fig. 1 and listed in Table 1. They are: 1) Dilute Single protein systems that contain a single copy of one of seven different transmembrane proteins having different sizes (proteins Pr1-Pr7, PrA, or PrB) with a lipid-to-protein (LP) ratio of 400. The nine single-protein systems are labeled from S1 to S7, and SA \& SB, where the last alphanumeric character refers to the protein type. 2) Crowded Monodisperse systems that contain nine copies of one protein type (Pr1-Pr7, PrA, or PrB) and 50 lipids per protein $(\mathrm{LP}=50)$. The nine crowded monodisperse systems are labeled from M1 to M7, and MA \& MB, where the last alphanumeric character refers to the protein type. 3) Polydisperse systems that contain simultaneously seven different protein types $(\operatorname{Pr} 1-\operatorname{Pr} 7)$ with a varying level of crowding. The number of copies of each protein type (1-10) is equal within every system, i.e., the most dilute system $(\mathrm{LP}=800)$ contains 1 copy of each protein (7 proteins in total) and the most crowded $(\mathrm{LP}=50)$ contains 10 copies of each protein (70 proteins in total). There are a total of six systems, labeled P50, P75, P100, P200, P400, and P800, where the number refers to the LP ratio. All polydisperse systems have approximately the same membrane surface area, i.e. the simulation boxes have the same lateral dimensions. 4) 2-dimensional Lennard-Jones (2DLJ) systems contain free particles with $\sigma=0.345 \mathrm{~nm}$ and disks that are formed by bonding together these free particles. Each simulated system contained 15 quasi-circular disks of various radii (from $0.4 \mathrm{~nm}$ formed by 7 particles bound together all the way to $7.6 \mathrm{~nm}$ formed as an aggregate of 1417 particles) at a certain particle-to-disk ratio that ranged from crowded (300 free particles per disk) to 
dilute (4000 free particles per disk) conditions. These five systems are labeled LJ300, LJ400, LJ600, LJ1000, and LJ4000, where the number refers to the free particle-to-disk ratio.

The first three sets of systems were simulated using the Martini model. ${ }^{31-33}$ Apart from the proteins, the membranes consisted of dipalmitoylphosphatidylcholine (DPPC) lipid and were solvated by water, $150 \mathrm{mM}$ of $\mathrm{NaCl}$, and counter-ions. However, the protein-protein interactions were reduced to avoid the irreversible and excessive aggregation of proteins. ${ }^{30,34}$ Simulations were performed under NPT conditions at $315 \mathrm{~K}$ using the standard Martini simulation parameters. The 2-dimensional Lennard-Jones model used in the fourth set successfully captured the qualitative behavior of crowded membranes in our earlier works. ${ }^{18,30,35}$ Further methodological details can be found in our earlier work. ${ }^{30}$ The simulation data for the polydisperse membranes P50-P400 are available at DOI: 10.5281/zenodo.846428 and that for the P800 membrane at DOI: 10.5281/zenodo.3465477. Data for monodisperse membranes are at DOI: 10.5281/zenodo.3572241, and for single-protein systems at DOI: 10.5281/zenodo.3572299. The first $1 \mu \mathrm{s}$ of all simulations was discarded from the analyses, and all simulations were performed using GROMACS v.5.0.x or later. ${ }^{36}$

To calculate the rotational diffusion coefficients, the GROMACS gmx rotmat tool was first used to extract the rotation matrix required for least squares fitting a conformation onto a reference one. Then, the projection of the rotation matrix to the membrane plane $(x-y$ plane in our simulation setup) was calculated, and the rotation angle in this plane, $\theta$, was calculated as a function of simulation time and corrected for the periodicity of rotation. A similar choice of plane was also used in Ref. 37. For large proteins that show little tilting, the rotation around membrane normal coincides with the rotational around their axis of inertia. However, the smallest proteins in our study display tilt angles of $\sim 20$ degrees, and therefore the rotational diffusion coefficients calculated here for small proteins may differ from the ones around intertia axes. Nevertheless, we consider the rotation around membrane normal to be the key parameter for protein oligomerization, and thus report rotational diffusion coefficients measured around this axis. Next, we calculated the time-averaged mean squared 
deviation of the rotation angle (MSDA) of $\theta$ as a function of lag time $\Delta$ as

$$
\overline{\delta_{\text {rot }}(\Delta)}=\frac{1}{T-\Delta} \int_{0}^{T-\Delta}[\theta(t+\Delta)-\theta(t)]^{2} \mathrm{~d} t
$$

for each copy of the proteins. Here $T$ is the total measurement time. The rotational diffusion coefficients of membrane proteins around the membrane normal, $D_{\text {rot }}$, were then calculated by fitting the MSDA data with

$$
\overline{\delta_{\text {rot }}(\Delta)}=2 D_{\text {rot }} \Delta^{\alpha}
$$

after reaching normal rotational diffusion, $\alpha=1$. When multiple copies of the same protein type are present in a membrane, we also averaged the $D_{\text {rot }}$ values over identical proteins. The standard deviation of these equivalent values serves as an error estimate. The MSDA data, shown for all proteins in the polydisperse systems in Fig. S1 in the Supporting Information (SI), demonstrate that normal rotational diffusion $(\alpha=1)$ is observed at $\Delta$ values between $100 \mathrm{~ns}$ and $1 \mu \mathrm{s}$ except for the most crowded conditions. This interval was hence used for fitting for all CG systems. The choice is limited by anomalous diffusion at short lag times and the lack of decent statistics at long ones. In Fig. S2, we provide a plot of MSDA/ $\Delta$ as a function of $\Delta$ to point out the possible deviations from normal diffusion and to demonstrate the quality of the fits. From Figs. S1 and S2, anomalous diffusion seems to persist into the time regime of dozens of nanoseconds for smaller proteins even in dilute systems, whereas larger proteins show normal diffusion already at a lag time of 10 ns, a topic we aim to clarify in the future. Notably, the time scales to reach normal diffusion for membrane proteins in this work are substantially longer than in previous simulations of water-soluble proteins where normal rotational diffusion was reached already after approximately $1 \mathrm{~ns} .{ }^{38}$

The values of effective radii describing the protein and its dynamically coupled lipid shell were taken from our earlier study. ${ }^{30}$ The rotational diffusion coefficients of singleprotein (S1-S7, SA, SB) and monodisperse (M1-M7, MA, MB) systems were corrected for 
finite size effects by dividing the diffusion coefficients by $\left(1-A_{\mathrm{p}} / A_{\mathrm{b}}\right)$, where $A_{\mathrm{b}}$ and $A_{\mathrm{p}}$ are the cross-sectional areas of the entire membrane and the protein, respectively. ${ }^{37}$ The latter was calculated considering cylindrical proteins with their effective radii. For polydisperse systems (P50-P800), the correction was negligible due to large membrane sizes and was hence omitted.

For 2DLJ systems, the MSDA data were fitted between 20 and 200 ns to extract $D_{\text {rot }}$. Fig. S3 demonstrates that the MSDA curves are linear in this fitting interval and the simulations capture normal diffusion. Curiously, we found out that the four smallest disks seemed to always rotate at the same speed, which was due to them rotating too fast for a reliable MSDA calculation at the used trajectory writing rate. Hence, we omitted these smallest disks and consider only the data for the other 11 ones in systems with different crowding levels.

\section{Results and Discussion}

We first analyzed the effects of crowding on rotational diffusion by calculating the $D_{\text {rot }}$ values of different proteins as a function of protein-to-lipid ratio from the polydisperse (P50-P800) systems. The slowing-down of rotational diffusion due to crowding is exponential in Fig. 2 (notice the logarithmic scale on the ordinate), and rotational motion seems to be affected by crowding more drastically than $D_{\text {lat }} \cdot{ }^{30}$ Interestingly, this agrees qualitatively with a very recent study on protein dynamics in solution. ${ }^{39}$ In STEPR experiments, the $D_{\text {rot }}$ of rhodopsin was found to decrease by $50 \%$ once crowding was increased form $\mathrm{LP}=150$ to $\mathrm{LP}=50 .{ }^{21}$ This agrees well with the behavior of our approximately similarly-sized Pr5, for which we extrapolated the diffusion to slow down by a factor of 2.2 over the same change in LP. However, another STEPR study revealed a very strong slowing-down effect for sarcoplasmic reticulum Ca-ATPase when the LP was decreased from 400 to $200 .{ }^{20}$ The observed 17 -fold slowdown is likely affected by an increased tendency of the peptides to dimerize. Because 


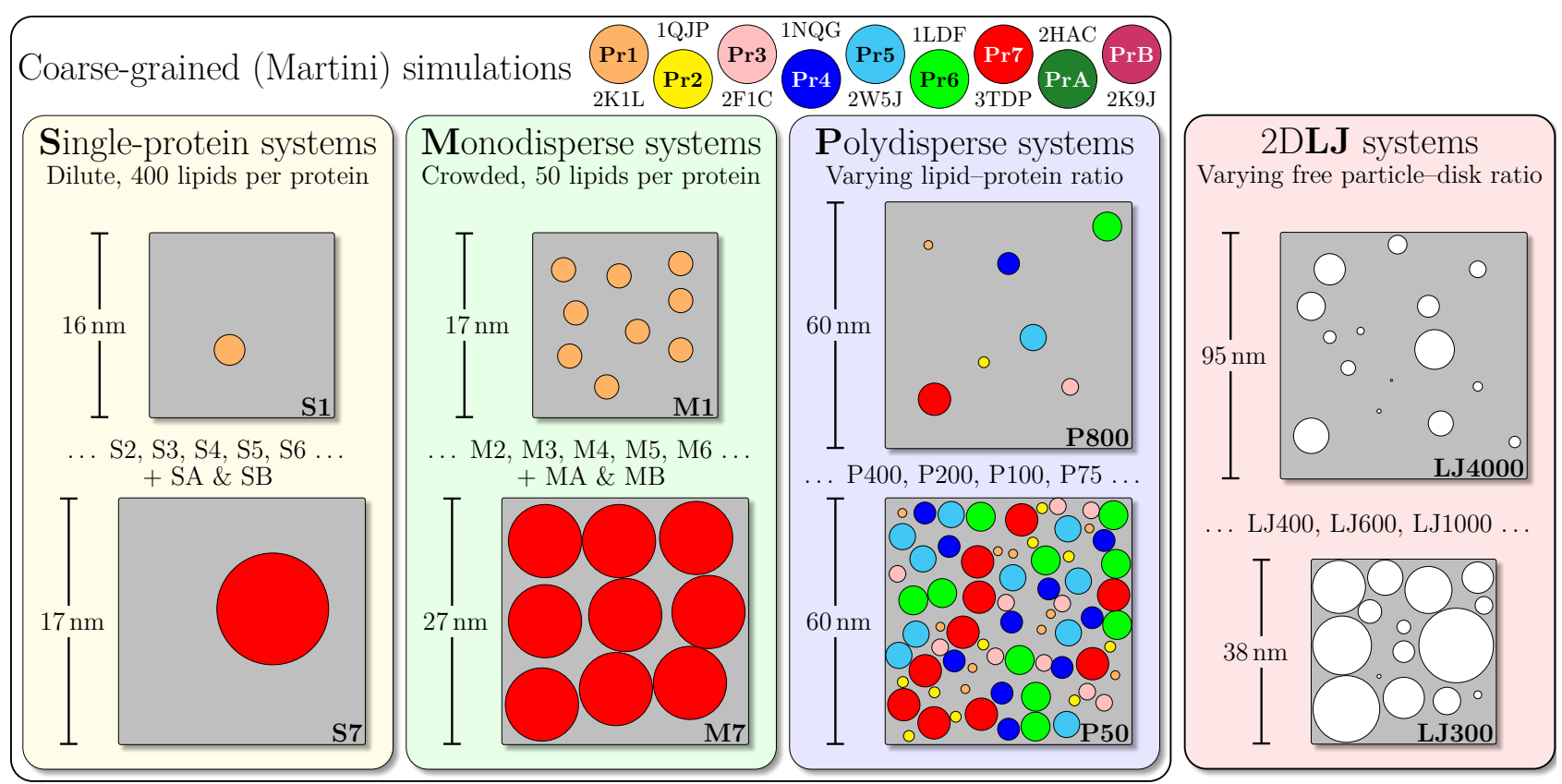

Figure 1: Schematic of the used simulation models. Three leftmost columns show coarsegrained simulations of proteins in lipid membranes using the Martini force field. The PDB identifiers of the proteins are given at the top of the schematic. The proteins are: $\operatorname{Pr} 1$ (2K1L): TM domain of the receptor tyrosine kinase EphA1; Pr2 (1QJP): Outer membrane protein A (OmpA) TM domain; Pr3 (2F1C): Outer membrane protein G (OmpG) porin; Pr4 (1NQC): Outer membrane cobalamin transporter (BtuB); Pr5 (2W5J): C14 rotor ring of the proton-translocating chloroplast ATP synthase; Pr6 (1LDF): Aquaglyceroporin (GlpF); Pr7 (3TDP): Hydrosulphide ion channel; PrA (2HAC): $\zeta$ TM domain of the T cell receptor; and $\operatorname{PrB}(2 \mathrm{~K} 9 \mathrm{~J})$ : Integrin $\alpha \mathrm{IIb}$ TM domain. Pr1, PrA, and PrB are $\alpha$-helices, $\operatorname{Pr} 2-\operatorname{Pr} 4$ are $\beta$-barrels, and Pr5-Pr7 are bundles of $\alpha$-helices. The rightmost column shows the 2dimensional simulations of the Lennard-Jones fluid with embedded disks. The labeling of the systems corresponds to that in Table 1, and the color coding of the proteins is consistent throughout all plots. Protein area coverage is accurate in the membrane depictions.

such protein-protein interactions are specific to particular proteins and dimerization is not observed in our simulations, we focus here on the changes in viscosity and excluded volume due to crowding only. This is achieved by the reduced protein-protein interactions.

While the qualitative effect of crowding on the rotational diffusion coefficients seems to be in line with experiments in our simulations, the rotational diffusion coefficient of single alpha helix (Pr1) in our coarse grained simulations of the P800 system, $150 \times 10^{5} \mathrm{rad}^{2} / \mathrm{s}$, is an order of magnitude larger than $8.0 \times 10^{5} \mathrm{rad}^{2} / \mathrm{s}$ estimated from the SD model and NMR 
Table 1: Table of simulated systems. "System" has the name of the system, "\# Prot." the number of proteins in the simulation, "LP" the lipid-to-protein ratio, and $T$ the simulation time. The notation $\operatorname{Pr}[1-7]$ refers to all seven proteins and $\mathrm{D}[1-15]$ to all the 15 disks.

\begin{tabular}{|c|c|c|c|}
\hline System & \# Prot. & LP & $T$ \\
\hline \multicolumn{4}{|c|}{ Polydisperse systems (6 membranes): } \\
\hline $\mathrm{P} 50$ & $10 \times \operatorname{Pr}[1-7]$ & 50 & $100 \mu \mathrm{s}$ \\
\hline P75 & $8 \times \operatorname{Pr}[1-7]$ & 75 & $100 \mu \mathrm{s}$ \\
\hline P100 & $6 \times \operatorname{Pr}[1-7]$ & 100 & $100 \mu \mathrm{s}$ \\
\hline P200 & $4 \times \operatorname{Pr}[1-7]$ & 200 & $100 \mu \mathrm{s}$ \\
\hline P400 & $2 \times \operatorname{Pr}[1-7]$ & 400 & $100 \mu \mathrm{s}$ \\
\hline P800 & $1 \times \operatorname{Pr}[1-7]$ & 800 & $51 \mu \mathrm{s}$ \\
\hline \multicolumn{4}{|c|}{ Monodisperse systems (9 membranes) } \\
\hline M1 & $9 \times \operatorname{Pr} 1$ & 50 & $20 \mu \mathrm{s}$ \\
\hline \multicolumn{4}{|c|}{ Identical for M2 - M7, MA, and MB } \\
\hline \multicolumn{4}{|c|}{ Single-protein systems (9 membranes) } \\
\hline S1 & $1 \times \operatorname{Pr} 1$ & 400 & $20 \mu \mathrm{s}$ \\
\hline \multicolumn{4}{|c|}{ Identical for S2 - S7, SA, and SB } \\
\hline \multicolumn{4}{|c|}{ 2DJL systems (6 "membranes"): } \\
\hline LJ4000 & $1 \times \mathrm{D}[1-15]$ & 4000 & $10 \mu \mathrm{s}$ \\
\hline LJ1000 & $1 \times \mathrm{D}[1-15]$ & 1000 & $50 \mu \mathrm{s}$ \\
\hline LJ600 & $1 \times \mathrm{D}[1-15]$ & 600 & $50 \mu \mathrm{s}$ \\
\hline LJ400 & $1 \times \mathrm{D}[1-15]$ & 400 & $50 \mu \mathrm{s}$ \\
\hline LJ300 & $1 \times \mathrm{D}[1-15]$ & 300 & $50 \mu \mathrm{s}$ \\
\hline
\end{tabular}

experiments for a single alpha helix. ${ }^{27}$ Rotational dynamics of proteins in atomistic MD simulations in solution was previously compared directly with NMR spin relaxation experiments detecting motions of backbone $\mathrm{N}-\mathrm{H}$ bonds, ${ }^{38}$ but this is not feasible here because atoms are not explicitly modelled in coarse grained simulations. Therefore, we estimate the slowest timescales corresponding a rotation of rigid body in lamellar geometry, $\tau=\left(D_{\text {rot }}\right)^{-1},{ }^{40}$ and compare these values to the timescales estimated from NMR experiments. This timescale is approximately $60 \mathrm{~ns}$ for a single alpha helix $(\operatorname{Pr} 1)$ in our simulation, which is significantly faster than $200 \mathrm{~ns}$ estimated for a single alpha helix from NMR experiments. ${ }^{26}$ Comparison 


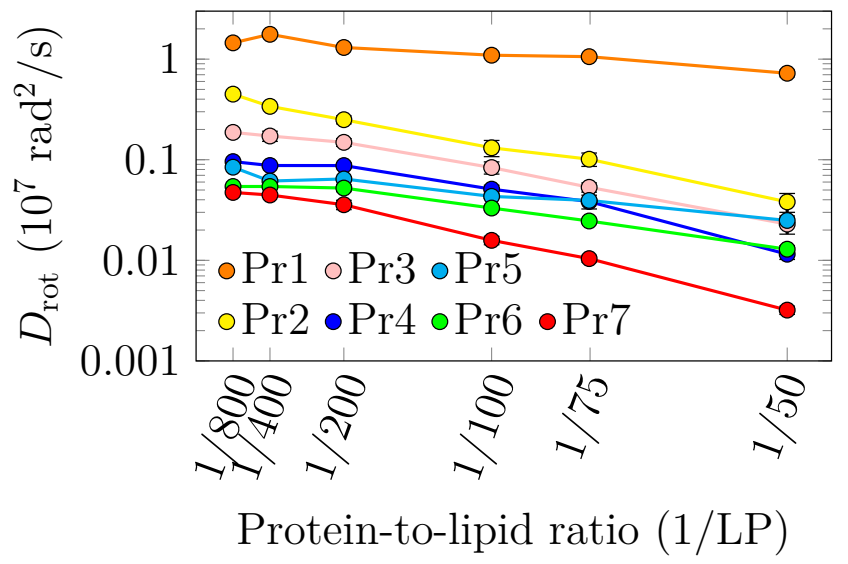

Figure 2: Rotational diffusion coefficients of the studied transmembrane proteins in different polydisperse systems, i.e. as a function of membrane crowding. Error bars, barely visible, show standard deviation within identical proteins. Note that the abscissa and ordinate are shown in linear and logarithmic scales, respectively.

of rotational motion of lipids between experiment and simulation provides similar result: the effective correlation time calculated for the vector connecting the two glycerol beads in our MARTINI POPC simulations, available at DOI: 10.5281/zenodo.3463130, was $0.3 \mathrm{ns,}$ which is an order of magnitude shorter than the experimental values for glycerol backbone 1.5-3.0 ns. ${ }^{41}$ In conclusion, the rotational dynamics in membranes is likely overestimated in the MARTINI model, similarly to lateral diffusion. ${ }^{42}$ Therefore, the effect of crowding on rotational diffusion should be estimated using the relative influence of crowding from our coarse grained simulations rather than the actual values of $D_{\text {rot }}$. For example, Cady et al. estimated from SD model that rotation of proteins with radius of appoximately $2.5 \mathrm{~nm}$ is sufficiently fast to measure $\mathrm{C}-\mathrm{H}$ and $\mathrm{N}-\mathrm{H}$ dipolar couplings, and ${ }^{15} \mathrm{~N}$ and ${ }^{13} \mathrm{C}$ chemical shift anisotropies. ${ }^{14}$ However, in our results crowding can reduce the rotational diffusion of protein with this size (Pr3) by an order of magnitude, which would decrease the rotation diffusion rate below the NMR interactions threshold in all the above mentioned experiments. ${ }^{14}$ Such a correction may have paramount importance when performing NMR experiments in native cell membranes. ${ }^{43}$

To test the validity of SD model for rotational diffusion in different crowding conditions, 
we plot the protein diffusion coefficients as a function of radii from all three sets of our CG MARTINI simulations in Fig. 3. For the polydisperse systems only data for the the representative P50, P400 and P800 systems are shown, while Fig. S4 contains the data for all polydisperse systems. In the most dilute simulations with a single protein (top panel in Fig. 3), the rotational diffusion coefficients follow the SD-like scaling of $D_{\text {rot }} \sim R^{-2}$ from Eq. (1). However, already in the very dilute polydisperse system with 800 lipids per protein, slight deviations from the SD model are observed. (P800 in Fig. 3). The systems with 400 and 200 lipids per protein (P400 \& P200 in Fig. 3 and Fig. S4) then correspond to a stronger scaling law of $D_{\text {rot }} \sim R^{-3}$. The polydisperse system with the highest crowding level (P50, 4th panel from the top in Fig. 3), probably corresponding to real cellular membranes, exhibits even stronger scaling. The same conclusions can be drawn from a plot of $D_{\text {rot }} \times R^{2}$ versus $R$ (see top panel of Fig. S6), where linear behavior is observed only for the singleprotein systems. Moreover, the scaling exponents $\nu$ from the fits in the form of $D_{\text {rot }} \sim R^{-\nu}$ are shown in the bottom panel of Fig. S6. They also demonstrate SD-like scaling $(\nu \approx 2)$ only for the single protein simulations, whereas the scaling exponent becomes systematically more negative upon crowding. Finally, we verified that the observed change in the scaling law was not only due to change in the tilt angle of proteins, which was significantly larger for single-pass proteins (Pr1, PrA, and PrB) than for larger proteins. Indeed, the fits of the scaling laws to $D_{\text {rot }}$ values without the single-pass proteins in Fig. S5 in the SI lead to the same conclusions as those presented in Fig. 3.

To study the generality of crowding-induced transition between the SD-like and stronger scaling laws ( $D_{\text {rot }} \sim R^{-\nu}$ with $\nu>2$ ), we calculated the rotational diffusion coefficients as a function of disk radius also from 2D LJ simulations (Fig. 4). In dilute conditions (LJ4000, top panel), the SD model (Eq. (3)) with $D_{\text {rot }} \sim R^{-2}$ scaling describes the data accurately. In systems with increasing crowding levels (panels towards the bottom), the SD model fits the data for small disks, while larger disks exhibit a stronger $D_{\text {rot }} \sim R^{-3}$ scaling. Only at the extreme crowding level (LJ300), the scaling seems to be at the edge of being stronger 


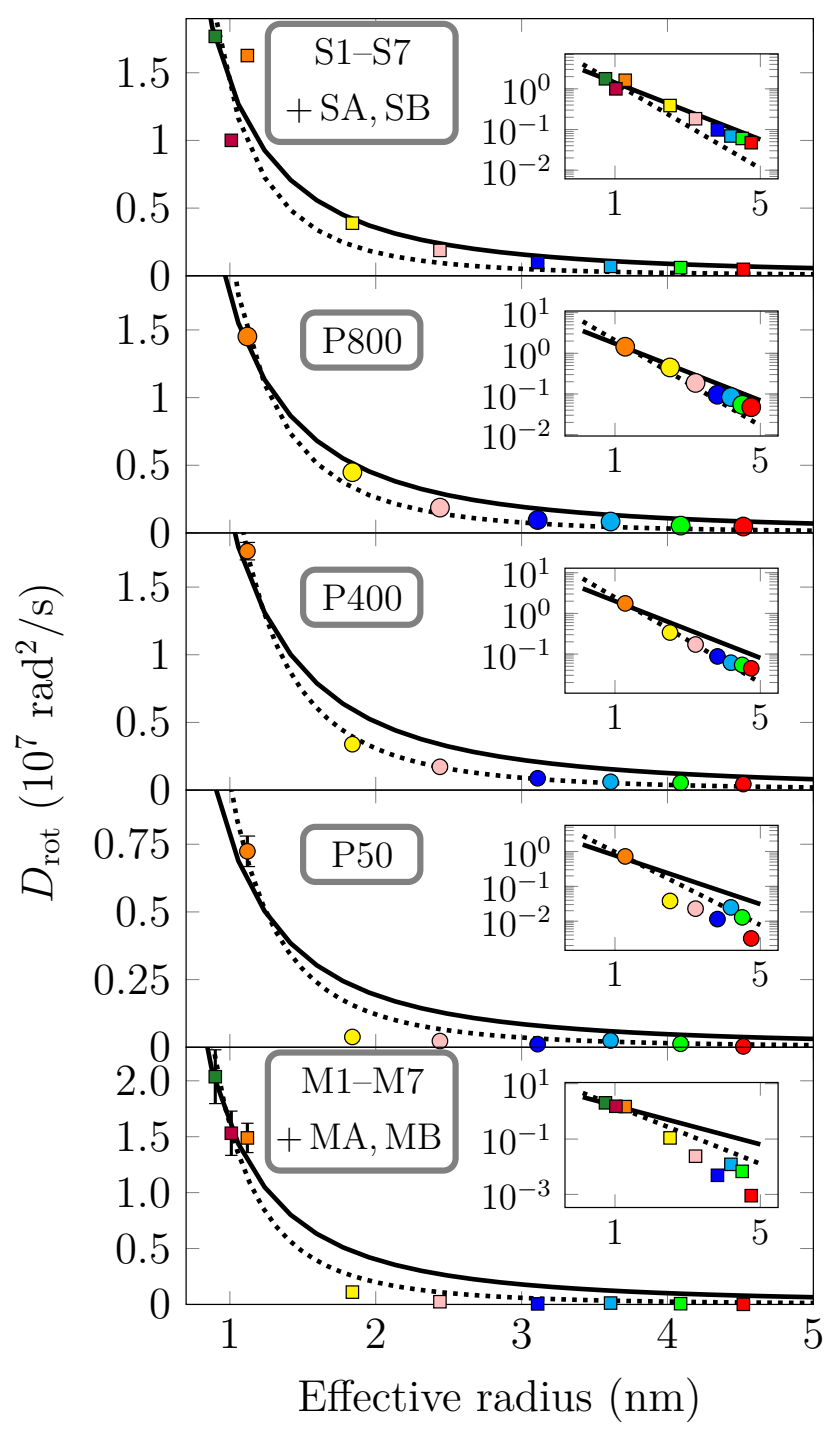

Figure 3: Rotational diffusion coefficients of the studied transmembrane proteins plotted as a function of their effective radii. The insets show the same data in $\log -\log$ scale. The solid line fit corresponds to the SD model $\left(D_{\text {rot }} \sim R^{-2}\right)$, while the dashed line shows the stronger scaling of $\left(D_{\text {rot }} \sim R^{-3}\right)$. Data are shown for the nine dilute single-protein systems (S1-S7, SA, SB in the top panel), for the seven proteins in three polydisperse membranes (dilute P800, P400, and crowded P50, three middlemost panels), and for the nine crowded monodisperse systems (M1-M7, MA, MB in the bottom panel). The squares show data corrected for finite size effects, ${ }^{37}$ whereas the circles are extracted from large systems where such effects play no role.

than $\nu>3$, in line with the CG systems (Fig. 3). The $D_{\text {rot }} \sim R^{-2}$ scaling holds up to a certain crossover radii, which is also evidenced by Fig. S7, where $D_{\text {rot }} \times R^{-2}$ is plotted against protein radii. The stronger scaling in the Martini CG systems is probably observed because 
proteins engage in transient oligomers even with the reduced protein-protein interactions. ${ }^{34}$ Because such oligomerization does not take place in our LJ systems, the different behavior between CG and LJ systems highlights the importance of specific interactions between a protein and its surroundings on its dynamics.

Notably, extensions of the SD model ${ }^{9-11}$ predict the $D_{\text {rot }} \sim R^{-3}$ scaling for inclusions with a radius larger than $L_{\mathrm{SD}}$. The regions of radius corresponding to the $D_{\text {rot }} \sim R^{-2}$ and $D_{\text {rot }} \sim R^{-3}$ scaling laws are highlighted by blue and red shading, respectively, in the insets in Fig. 4. Interestingly, the crossover radius shifts toward smaller values upon increase in crowding. This suggests that either the value of $L_{\mathrm{SD}}$ decreases upon crowding, or that the hydrodynamic radii of the disks increase. In addition to the membrane viscosity, $L_{\mathrm{SD}}$ depends also on the solvent viscosity and membrane thickness, but these are neither present nor well defined for the 2DLJ system. This indicates that either the bulk LJ fluid viscosity decreases upon crowding or that the hydrodynamic radii of the inclusions increases and possibly compensates for an increased membrane viscosity. Another option is that - not surprisingly - the SD does not provide a proper description of the observed dynamics in the 2-dimensional system.

To unravel the reasons of the observed shift to stronger scaling, let us discuss the CG systems where all the aforementioned parameters are well-defined. The CG simulations display a similar crossover between SD-like and a stronger scaling law, yet unlike for the 2DLJ data, no clear crossover at a certain radius value is observed. This is likely due to three factors: the number of proteins is limited in our study, the size range of existing (and hence simulated) membrane proteins is fairly limited, and our CG data are somewhat noisy due to complex interactions in crowded systems. To analyze if the stronger scaling could be explained by the protein radii being larger than the SD length, we calculated $L_{\mathrm{SD}}$ under the most dilute (P800) conditions by fitting the lateral diffusion coefficients of proteins with different radii with the SD model for lateral diffusion. However, all of the simulated proteins are smaller than the estimated a value of $\sim 13.9 \mathrm{~nm}$ for $L_{\mathrm{SD}}$. 


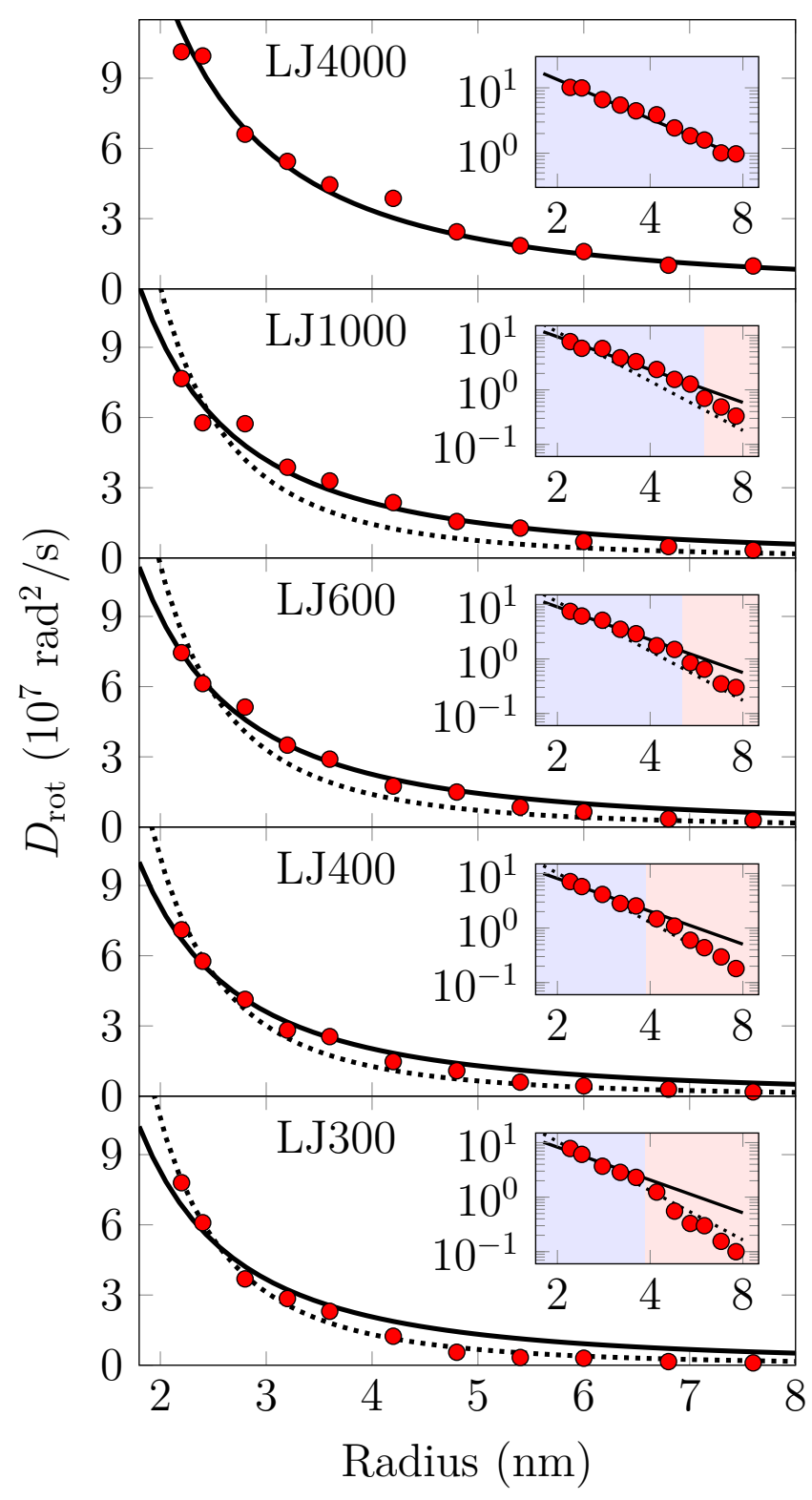

Figure 4: Rotational diffusion coefficients of the disks as a function of their radii in the 2-dimensional LJ systems. The solid line fit corresponds to the SD model $\left(D_{\text {rot }} \sim R^{-2}\right)$, while the dashed line shows the stronger scaling of $\left(D_{\text {rot }} \sim R^{-3}\right)$. For the uppermost panel, the latter fit could not be performed in a reasonable manner. The insets show the data in $\log -\log$ scale, and the blue and red shadings correspond to regions better described by the $D_{\text {rot }} \sim R^{-2}$ and $D_{\text {rot }} \sim R^{-3}$ fits, respectively. Note that the fitting is always performed across all radii.

The SD length further increases with crowding level, because viscosity increases upon crowding from $5.0 \mathrm{mPa} \cdot \mathrm{s}$ for dilute (single-protein) system to $13.5 \mathrm{mPa} \cdot \mathrm{s}$ for crowded (LP=50) 
membranes (see SI for details on the calculation). This is in line with the observed decrease of both rotational (Fig. 2) and lateral diffusion coefficients (Fig. 2 in Ref. 30). While it seems that the viscosity observed by rotational and translational motion might not always be equal in living cells, ${ }^{25}$ our simulations do not include the cellular complexity, such as the cytoskeleton, which is likely behind this difference. Indeed, the aforementioned viscosity value of $5.0 \mathrm{mPa} \cdot \mathrm{s}$, obtained from lateral motion, agrees favorably from a value of $4.7 \mathrm{mPa} \cdot \mathrm{s}$ obtained from a fit of the SD model to rotational diffusion in single-protein systems (top panel in Fig. 3). Alternatively, the effective radius of proteins could increase with crowding, but this was not the case for Pr3 protein in Fig. S6 in the Supporting Information of our previous work, ${ }^{30}$ where we studied the effect of the lipid-to-protein ratio on the effective radius in single-protein systems. Therefore, we cannot explain the shift to the stronger scaling by the increase of protein radius above the SD length. One possible reason not considered in this work is the emergence of correlated movements between nearby proteins in the more crowded systems, which requires a closer look in future studies. Unfortunately, our data were too noisy for us to verify such hypothesis. We also verified that the wobbling of the proteins is fairly small (see Fig. S8 in the SI) and does not hence compromise the calculation of the rotational angle, which might lead to a stronger scaling observed for 3-dimensional rotation diffusion.

After this careful examination of possible factors explaining the crossover from $D_{\text {rot }} \sim R^{-2}$ to a stronger scaling law, we cannot but conclude that protein crowding seems to lead to the breakdown of the SD description of rotational motion. In our recent work, we reached a similar conclusion also in terms of lateral diffusion. ${ }^{30}$ These findings are important for experiments where information on protein size or membrane properties are deduced from diffusion behavior in crowded environments — such as the membranes of cells. ${ }^{16}$ 


\section{Conclusions}

Concluding, we analyzed the rotational motion of various proteins and protein-like inclusions in membranes using extensive simulation data spanning different levels of protein concentrations from dilute membranes to realistically-crowded ones. We found that the rotational diffusion rates of proteins decrease approximately exponentially with increasing number of proteins with respect to membrane lipids. Only at very dilute conditions, rotational diffusion follows the SD model and scales as $D_{\text {rot }} \sim R^{-2}$. However, even at fairly dilute conditions compared to biologically relevant systems, a stronger $D_{\text {rot }} \sim R^{-3}$ scaling is observed. When protein concentration is increased to levels characteristic for cellular membranes, the dependency of $D_{\text {rot }}$ on $R$ becomes even stronger. Our synthetic 2-dimensional LJ simulations do not capture the stronger than $D_{\text {rot }} \sim R^{-3}$ scaling, indicating that it arises due to specific protein-protein interactions. Therefore, our observations suggest that the SD model should be employed with care to estimate protein size or membrane properties, such as viscosity, in living cells or plasma membrane-derived membranes. Moreover, since the reduced rate of rotational motion upon crowding may influence the detectability of interactions in NMR experiments, ${ }^{14}$ it might be better to consider the $D_{\text {rot }} \sim R^{-3}$ or stronger scaling when estimating the detection limits in native membranes.

The findings of this work — together with our previous study — suggest that protein

oligomerization processes with paramount implications in protein function ${ }^{3,4}$ depend very strongly on protein size: Not only do lateral diffusion coefficients scale as $D_{\text {lat }} \sim R^{-1}$ suggesting that larger proteins are fairly stationary in cellular scale as as smaller proteins rapidly sample the membrane plane, ${ }^{30}$ but also the rotational diffusion of these large proteins is slower than expected from the established SD model. This provides advantage for proteins with small transmembrane segments to form oligomers through improved the sampling of dimerization intefaces. These findings might even explain why many receptors — such as the tyrosine kinases — that need to dimerize for their proper function, contain only a single transmembrane helix. ${ }^{44}$ 


\section{Acknowledgement}

We thank CSC-IT Center for Science for computational resources. M.J. thanks Emil Aaltonen foundation for funding, and O.H.S.O. acknowledges financial support from Academy of Finland (315596). M.J. and H.M.-S. acknowledge Czech Science Foundation (EXPRO grant 19-26854X).

\section{Supporting Information Available}

Details on the calculation of membrane viscosity. Additional results.

\section{References}

(1) Enkavi, G.; Javanainen, M.; Kulig, W.; Róg, T.; Vattulainen, I. Multiscale Simulations of Biological Membranes: the Challenge to Understand Biological Phenomena in a Living Substance. Chem. Rev. 2019, 119, 5607-5774.

(2) Corradi, V.; Sejdiu, B. I.; Mesa-Galloso, H.; Abdizadeh, H.; Noskov, S. Y.; Marrink, S. J.; Tieleman, D. P. Emerging Diversity in Lipid-Protein Interactions. Chem. Rev. 2019, 119, 5775-5848.

(3) Gahbauer, S.; Böckmann, R. A. Membrane-Mediated Oligomerization of G Protein Coupled Receptors and Its Implications for GPCR Function. Front. Physiol. 2016, 7, 494.

(4) George, S. R.; O’Dowd, B. F.; Lee, S. P. G-Protein-Coupled Receptor Oligomerization and Its Potential for Drug Discovery. Nat. Rev. Drug Discov. 2002, 1, 808.

(5) Kuttner, Y. Y.; Kozer, N.; Segal, E.; Schreiber, G.; Haran, G. Separating the Contribution of Translational and Rotational Diffusion to Protein Association. J. Am. Chem. Soc. 2005, 127, 15138-15144. 
(6) Shoup, D.; Lipari, G.; Szabo, A. Diffusion-Controlled Bimolecular Reaction Rates. The Effect of Rotational Diffusion and Orientation Constraints. Biophys. J. 1981, 36, 697714.

(7) Saffman, P.; Delbrück, M. Brownian Motion in Biological Membranes. Proc. Natl. Acad. Sci. USA 1975, 72, 3111-3113.

(8) Saffman, P. Brownian Motion in Thin Sheets of Viscous Fluid. J. Fluid Mech. 1976, 73, 593-602.

(9) Petrov, E. P.; Schwille, P. Translational Diffusion in Lipid Membranes Beyond the Saffman-Delbrück Approximation. Biophys. J. 2008, 94, L41-L43.

(10) Petrov, E. P.; Petrosyan, R.; Schwille, P. Translational and Rotational Diffusion of Micrometer-Sized Solid Domains in Lipid Membranes. Soft Matter 2012, 8, 7552-7555.

(11) Hughes, B.; Pailthorpe, B.; White, L. The Translational and Rotational Drag on a Cylinder Moving in a Membrane. J. Fluid Mech. 1981, 110, 349-372.

(12) Peters, R.; Cherry, R. J. Lateral and Rotational Diffusion of Bacteriorhodopsin in Lipid Bilayers: Experimental Test of the Saffman-Delbrück Equations. Proc. Natl. Acad. Sci. USA 1982, 79, 4317-4321.

(13) Hormel, T. T.; Kurihara, S. Q.; Brennan, M. K.; Wozniak, M. C.; Parthasarathy, R. Measuring Lipid Membrane Viscosity Using Rotational and Translational Probe Diffusion. Phys. Rev. Lett. 2014, 112, 188101.

(14) Cady, S. D.; Goodman, C.; Tatko, C. D.; DeGrado, W. F.; Hong, M. Determining the Orientation of Uniaxially Rotating Membrane Proteins Using Unoriented Samples: a ${ }^{2} \mathrm{H},{ }^{13} \mathrm{C}$, and ${ }^{15} \mathrm{~N}$ Solid-State NMR Investigation of the Dynamics and Orientation of a Transmembrane Helical Bundle. J. Am. Chem. Soc. 2007, 129, 5719-5729. 
(15) Guigas, G.; Weiss, M. Effects of Protein Crowding on Membrane Systems. BBA Biomembranes 2016, 1858, 2441-2450.

(16) Dupuy, A. D.; Engelman, D. M. Protein Area Occupancy at the Center of the Red Blood Cell Membrane. Proc. Natl. Acad. Sci. USA 2008, 105, 2848-2852.

(17) Krapf, D.; Metzler, R. Strange Interfacial Molecular Dynamics. Phys. Today 2019, 72, 48-55.

(18) Jeon, J.; Javanainen, M.; Martinez-Seara, H.; Metzler, R.; Vattulainen, I. Protein Crowding in Lipid Bilayers Gives Rise to Non-Gaussian Anomalous Lateral Diffusion of Phospholipids and Proteins. Phys. Rev. X 2016, 6, 021006.

(19) Niemelä, P. S.; Miettinen, M. S.; Monticelli, L.; Hammaren, H.; Bjelkmar, P.; Murtola, T.; Lindahl, E.; Vattulainen, I. Membrane Proteins Diffuse as Dynamic Complexes with Lipids. J. Am. Chem. Soc. 2010, 132, 7574-7575.

(20) James, Z. M.; McCaffrey, J. E.; Torgersen, K. D.; Karim, C. B.; Thomas, D. D. ProteinProtein Interactions in Calcium Transport Regulation Probed by Saturation Transfer Electron Paramagnetic Resonance. Biophys. J. 2012, 103, 1370-1378.

(21) Kusumi, A.; Hyde, J. S. Spin-Label Saturation-Transfer Electron Spin Resonance Detection of Transient Association of Rhodopsin in Reconstituted Membranes. Biochemistry 1982, 21, 5978-5983.

(22) Ryba, N. J.; Marsh, D. Protein Rotational Diffusion and Lipid/Protein Interactions in Recombinants of Bovine Rhodopsin With Saturated Diacylphosphatidylcholines of Different Chain Lengths Studied by Conventional and Saturation-Transfer Electron Spin Resonance. Biochemistry 1992, 31, 7511-7518.

(23) Fajer, P.; Knowles, P. F.; Marsh, D. Rotational Motion of Yeast Cytochrome Oxi- 
dase in Phosphatidylcholine Complexes Studied by Saturation-Transfer Electron Spin Resonance. Biochemistry 1989, 28, 5634-5643.

(24) Cone, R. A. Rotational Diffusion of Rhodopsin in the Visual Receptor Membrane. Nature New Biol. 1972, 236, 39.

(25) Spille, J.-H.; Zürn, A.; Hoffmann, C.; Lohse, M. J.; Harms, G. S. Rotational diffusion of the $\alpha 2$ a Adrenergic Receptor Revealed by FlAsH Labeling in Living Cells. Biophys. J. 2011, 100, 1139-1148.

(26) Pauls, K. P.; MacKay, A. L.; Söderman, O.; Bloom, M.; Tanjea, A. K.; Hodges, R. S. Dynamic Properties of the Backbone of an Integral Membrane Polypeptide Measured by ${ }^{2}$ H-NMR. Eur. Biophys. J. 1985, 12, 1-11.

(27) Awosanya, E. O.; Nevzorov, A. A. Protein Rotational Dynamics in Aligned Lipid Membranes Probed by Anisotropic T1 $\rho$ NMR Relaxation. Biophys. J. 2018, 114, 392-399.

(28) Guigas, G.; Weiss, M. Size-Dependent Diffusion of Membrane Inclusions. Biophys. J. 2006, 91, 2393-2398.

(29) Goose, J. E.; Sansom, M. S. Reduced Lateral Mobility of Lipids and Proteins in Crowded Membranes. PLoS Comp. Biol. 2013, 9, e1003033.

(30) Javanainen, M.; Martinez-Seara, H.; Metzler, R.; Vattulainen, I. Diffusion of Integral Membrane Proteins in Protein-Rich Membranes. J. Phys. Chem. Lett. 2017, 8, 43084313.

(31) Monticelli, L.; Kandasamy, S. K.; Periole, X.; Larson, R. G.; Tieleman, D. P.; Marrink, S.-J. The MARTINI Coarse-Grained Force Field: Extension to Proteins. J. Chem. Theory Comput. 2008, 4, 819-834.

(32) de Jong, D. H.; Singh, G.; Bennett, W. D.; Arnarez, C.; Wassenaar, T. A.; Schäfer, L. V.; Periole, X.; Tieleman, D. P.; Marrink, S. J. Improved Parameters for 
the Martini Coarse-Grained Protein Force Field. J. Chem. Theory Comput. 2012, 9, 687-697.

(33) Marrink, S. J.; Risselada, H. J.; Yefimov, S.; Tieleman, D. P.; De Vries, A. H. The MARTINI Force Field: Coarse Grained Model for Biomolecular Simulations. J. Phys. Chem. B 2007, 111, 7812-7824.

(34) Javanainen, M.; Martinez-Seara, H.; Vattulainen, I. Excessive Aggregation of Membrane Proteins in the Martini Mmodel. PLoS One 2017, 12, e0187936.

(35) Jeon, J.-H.; Monne, H. M.-S.; Javanainen, M.; Metzler, R. Anomalous Diffusion of Phospholipids and Cholesterols in a Lipid Bilayer and Its Origins. Phys. Rev. Lett. 2012, 109, 188103.

(36) Abraham, M. J.; Murtola, T.; Schulz, R.; Páll, S.; Smith, J. C.; Hess, B.; Lindahl, E. GROMACS: High Performance Molecular Simulations Through Multi-Level Parallelism From Laptops to Supercomputers. SoftwareX 2015, 1, 19-25.

(37) Vögele, M.; Koefinger, J.; Hummer, G. Finite-Size Corrected Rotational Diffusion Coefficients of Membrane Proteins and Carbon Nanotubes from Molecular Dynamics Simulations. J. Phys. Chem. B 2019,

(38) Ollila, O. H. S.; Heikkinen, H. A.; Iwa Âr, H. Rotational Dynamics of Proteins from Spin Relaxation Times and Molecular Dynamics Simulations. J. Phys. Chem. B 2018, 122, 6559-6569.

(39) von Bülow, S.; Siggel, M.; Linke, M.; Hummer, G. Dynamic Cluster Formation Determines Viscosity and Diffusion in Dense Protein Solutions. Proc. Natl. Acad. Sci. USA 2019, 116, 9843-9852.

(40) Woessner, D. E. Nuclear Spin Relaxation in Ellipsoids Undergoing Rotational Brownian Motion. J. Chem. Phys. 1962, 37, 647-654. 
(41) Ferreira, T. M.; Ollila, O. H. S.; Pigliapochi, R.; Dabkowska, A. P.; Topgaard, D. Model-Free Estimation of the Effective Correlation Time for $\mathrm{C}-\mathrm{H}$ Bond Reorientation in Amphiphilic Bilayers: ${ }^{1} \mathrm{H}-{ }^{13} \mathrm{C}$ Solid-State NMR and MD Simulations. J. Chem. Phys. 2015, 142, 044905.

(42) Ramadurai, S.; Holt, A.; Schäfer, L. V.; Krasnikov, V. V.; Rijkers, D. T.; Marrink, S. J.; Killian, J. A.; Poolman, B. Influence of Hydrophobic Mismatch and Amino Acid Composition on the Lateral Diffusion of Transmembrane Peptides. Biophys. J. 2010, 99, 1447-1454.

(43) Baker, L. A.; Sinnige, T.; Schellenberger, P.; de Keyzer, J.; Siebert, C. A.; Driessen, A. J.; Baldus, M.; Grünewald, K. Combined ${ }^{1}$ H-Detected Solid-State NMR Spectroscopy and Electron Cryotomography to Study Membrane Proteins across Resolutions in Native Environments. Structure 2018, 26.

(44) Hubbard, S. R.; Till, J. H. Protein Tyrosine Kinase Structure and Function. Annu. Rev. Biochem. 2000, 69, 373-398. 


\section{Graphical TOC Entry}

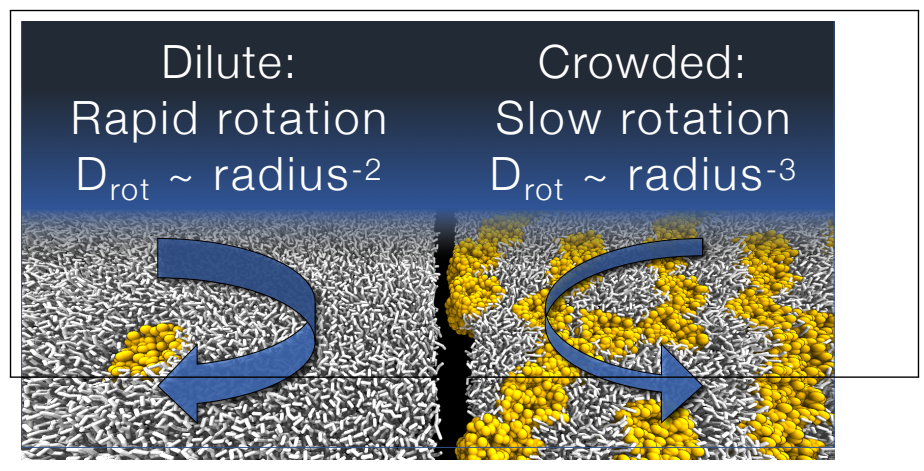

